\title{
Étude de poste et zonage aux blocs opératoires : méthodologie pratique en six étapes
}

\author{
C. Duverger ${ }^{1}$, G. Moliner ${ }^{1}$, F. Boury ${ }^{1}$, P. Branchereau ${ }^{2}$, P. Costa ${ }^{2}$, J.P. Beregi ${ }^{1}$ et J. Greffier ${ }^{1 \star}$ \\ ${ }^{1}$ CHU Carémeau de Nîmes, Service d'imagerie médicale, Place du Pr. Robert Debré, 30929 Nîmes Cedex 9, France. \\ 2 CHU Carémeau de Nîmes, Service de chirurgie, Place du Pr. Robert Debré, 30929 Nîmes Cedex 9, France.
}

Reçu le 5 mars 2015 - Accepté le 9 juillet 2015

\begin{abstract}
Résumé - La mise en place de la radioprotection aux blocs opératoires est longue et complexe. Les études de poste de travail et le zonage des locaux sont difficiles à réaliser. Une méthodologie a été mise en place au CHU de Nîmes depuis 2012. Cette méthodologie, composée de 6 étapes, permet de pallier les difficultés rencontrées. L'observation des pratiques (étape 1) permet de s'immerger dans l'environnement, les contraintes et l'activité du bloc opératoire, de s'intégrer aux équipes chirurgicales, d'identifier les actes les plus pénalisants et de mettre en place une base de données dosimétrique. L'étape de préparation (étape 2) consiste à regrouper les actes par type d'intervention et à choisir le matériel nécessaire aux mesures. Les simulations (étape 3) permettent d'évaluer par des mesures les différentes expositions aux différents postes de travail. L'étape d'extrapolation (étape 4) consiste à pallier le manque d'information et la complexité de l'activité. La réalisation (étape 5) du zonage et des études de postes est alors menée. Le suivi dans le temps (étape 6) et l'amélioration de la base de données dosimétriques doivent permettre de suivre l'évolution des pratiques et de réaliser l'optimisation des pratiques en lien avec le physicien médical. Cette méthodologie est applicable à tous les secteurs où sont effectués des actes médicaux guidés par radioscopie en l'adaptant à leur structure et à leur organisation.
\end{abstract}

\begin{abstract}
Occupational study and zoning in operational theatre: six-step practical methodology. The implementation of radiation protection in operating theatres is long and complex. Occupational studies and zoning are difficult. A methodology has been set up at the University Hospital of Nimes since 2012. This methodology, consisting of 6 steps, overcomes the difficulties. Observation of practices (step 1) allows familiarization with constraints and activity, integration of surgical teams, identification of the most penalizing procedures and establishment of a dosimetric database. Preparation (step 2) consists of grouping procedures by type of intervention and selecting the equipment needed for simulations. Simulations (step 3) allow us to obtain different exposures for different workstations. Extrapolation (step 4) is made to overcome the lack of information and the complexity of the activity. Zoning and occupational studies (step 5) are then conducted. Monitoring over time (step 6) and improving the dosimetric database enable us to follow the evolution of practices and achieve optimization in collaboration with the medical physicist. This methodology is applicable to all areas where fluoroscopically-guided medical procedures are performed by adapting to suit their structure and organization.
\end{abstract}

Keywords: interventional radiology / radiation risk / dose assessment / fluoroscopy / occupational dosimetry

\section{Introduction}

Avec l'augmentation des possibilités des techniques miniinvasives utilisant les rayonnements ionisants, l'utilisation des arceaux de blocs tend à augmenter dans les blocs opératoires et à se banaliser. Cette utilisation non maîtrisée par le personnel du bloc, nécessite d'être très attentif à la bonne application des principes de radioprotection, afin de réduire l'exposition des patients et des travailleurs à un niveau aussi bas que raisonnablement possible (ALARA).

La radioprotection dans les blocs opératoires est souvent considérée comme accessoire au vu des risques spécifiques

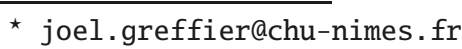

liés au geste invasif. Le personnel présent dans ce secteur est peu sensibilisé et la présence d'une personne compétente en radioprotection (PCR) issue du bloc est très rare. Les PCR sont le plus souvent issues d'autres services et rencontrent des difficultés à s'intégrer et à s'acclimater à cette activité. Cependant, comme pour tout autre secteur utilisant les rayonnements ionisants, de nombreuses tâches indispensables à la PCR et réglementaires doivent être réalisées dans les blocs opératoires.

La réglementation impose à l'employeur de procéder à la délimitation des zones de travail soumises aux rayonnements (JORF, 2006a) et à une analyse des poste de travail vis-à-vis de l'ensemble des conditions pouvant affecter la santé et la sécurité des travailleurs (JORF, 2003). Le zonage et l'étude 
Tableau 1. Ensemble des paramètres à relever lors de ces observations. All parameters measured during observations.

\begin{tabular}{ll}
\hline Intervenants & Indicateurs dosimétriques \\
\hline - Poste de travail (fonction) & - Produit Dose Surface (PDS) \\
- Distance par rapport au patient & - Temps de radioscopie \\
- Position debout ou assise & - Nombre d'images (si mode graphie utilisé) \\
Arceau de bloc & Paramètres d'exposition \\
\hline - Hauteur de la table de traitement & - Mode et type de radioscopie \\
- Région anatomique explorée & - Tension (kV) la plus utilisée \\
- Modèle, orientation et incidence & - Quantité de courant (mA) la plus utilisée \\
- Distance tube-patient et patient-détecteur & - Radiographie (si utilisée) \\
\hline
\end{tabular}

de poste posent souvent des problèmes de réalisation pratique (IRSN, 2010) dans les blocs opératoires (Schiedts, 2014). Les actes sont nombreux et variés. Les arceaux de blocs sont différents selon les constructeurs. Mais surtout, les intervenants sont en nombre conséquent et répartissent leurs activités sur plusieurs salles de façon variable en fonction des plannings.

En 2012, la démarche de radioprotection aux blocs opératoires a été revue au CHU de Nîmes. Les blocs opératoires de notre centre sont composés de 20 salles et disposent de 8 arceaux de bloc. Sept spécialités chirurgicales utilisant les rayonnements ionisants sont présentes : Orthopédie-Traumatologie, Neurochirurgie, ORL, Vasculaire, Urologie, Digestif et Gynécologie. En 2014, 19149 actes ont été réalisés dont 4387 avec utilisation d'arceaux de bloc. La cellule de radioprotection a réalisé le zonage et les études de poste de ce secteur en décomposant en différentes étapes essentielles : observations préparation - simulation - extrapolation - réalisation - suivi dans le temps.

L'objectif de notre étude est de fournir à chaque PCR une méthodologie structurée, pratique et basée sur 6 étapes indispensables, pour la réalisation des études de postes et zonages réglementaires de radioprotection aux blocs opératoires.

\section{2 Étape 1 : observations}

La première étape indispensable à toutes tâches de radioprotection consiste à observer et comprendre les pratiques. L'observation des actes en salle doit permettre d'identifier l'ensemble des postes de travail auxquels sont exposées les différentes catégories d'agents.

\subsection{S’approprier l'activité}

L'activité des blocs opératoires se divise entre plusieurs spécialités. Dans chaque spécialité, des actes sont réalisés avec et sans arceaux de blocs. Pour pouvoir quantifier les actes avec rayonnements ionisants, un classement doit être réalisé. Une base de données doit alors être mise en place. Celle-ci comporte la liste des différents actes réalisés aux blocs opératoires et l'ensemble des personnels présents en salle.

Pour chaque libellé d'acte, trois critères d'utilisation des rayonnements ionisants sont définis : systématique, occasionnelle ou sans rayonnements ionisants. Ce classement est réalisé en concertation avec les différentes équipes.
Tableau 2. Postes de travail et catégories d'agent des blocs opératoires.

Workstations and categories of agent operating theaters.

\begin{tabular}{lc}
\hline Poste & Catégorie \\
\hline Chirurgien $(\mathrm{C})$ & Chirurgien ou interne \\
Aide opératoire $(\mathrm{O})$ & Chirurgien, interne ou IBODE \\
Instrumentiste $(\mathrm{I})$ & IBODE \\
Circulant $(\mathrm{L})$ & IBODE \\
Anesthésie $(\mathrm{A})$ & Médecin ou IADE \\
MERM $(\mathrm{M})$ & MERM \\
\hline
\end{tabular}

IBODE : Infirmier de Bloc Opératoire Diplômé d'Etat ; IADE : Infirmier Anesthésiste Diplômé d'Etat; MERM : Manipulateur en Electroradiologie Médicale.

\subsection{S’imprégner et apprendre}

Une fois le recueil des actes réalisé, la PCR doit sélectionner les actes avec rayonnements ionisants les plus réalisés par spécialité et se rendre en salle opératoire pour assister à ces actes. Cette étape permet à la PCR de s'intégrer aux équipes médicales et paramédicales et de s'imprégner de toutes leurs contraintes. L'observation doit permettre de relever pour chaque acte : les différents intervenants, le positionnement de l'arceau de bloc, les indicateurs dosimétriques et les constantes d'exposition (Tableau 1). Cependant, les observations en salle prennent un temps conséquent et ne permettent pas de suivre l'ensemble de l'activité chirurgicale.

\subsection{Se projeter et débriefer}

À partir des observations réalisées en salle, la PCR peut ensuite définir les différents postes de travail pouvant être occupés par différentes catégories d'agent (Tableau 2). Chaque acte fait l'objet d'un plan à l'échelle reproduisant la position du matériel et des agents la plus fidèle à l'acte observé (Figure 1). Ces plans sont présentés et validés par les équipes des différents secteurs.

\subsection{Recueil et enregistrement des indicateurs dosimétriques}

Pour pouvoir débuter les mesures nécessaires à la réalisation des zonages et des analyses de postes de travail, la PCR 
doit avoir à disposition une base de données suffisamment représentative de l'activité réelle. Ce registre doit comporter a minima les libellés d'acte, la spécialité et les indicateurs dosimétriques. Ces indicateurs sont parfois relevés par le personnel des blocs (cahier, feuille de suivi, saisie informatique). À défaut, la PCR peut les relever via l'impression des rapports de dose sur les différents arceaux. Ce recueil doit être démarré au plus tôt et être le plus précis et complet possible. La mise en place de cette base de données permet d'avoir une vision plus précise des pratiques et confirme la sélection des actes effectuée initialement. Le recueil et l'enregistrement des indicateurs dosimétriques sont par ailleurs utiles à la personne spécialisée en radiophysique médicale (PSRPM) pour assurer un suivi (JORF, 2006b) et une optimisation des doses délivrées aux patients (JORF, 2011).

\subsection{Synthétiser}

Une fois la phase d'observations achevée, la PCR connait plus spécifiquement l'environnement, la complexité et les contraintes liées aux blocs opératoires. Les positions des agents aux postes de travail, les roulements des équipes et les activités sont définis. Les actes les plus réalisés et les plus irradiants sont identifiés. L'étape suivante consiste à préparer les simulations des actes observés.

\section{3 Étape 2 : préparation}

Lors de l'étape de préparation, la PCR procède au regroupement des actes et choisit le matériel qui lui sera nécessaire en vue des simulations.

\subsection{Regroupement des actes}

Les actes sont regroupés par spécialité et par type d'intervention en fonction des paramètres observés (Tableau 1). Pour chaque spécialité, 1 à 5 types d'interventions sont définis afin d'être les plus représentatifs possible de l'activité.

Un type d'intervention peut être commun à plusieurs spécialités (mise en place d'un cathéter à chambre implantable (PAC)) et une disposition (Figure 1) commune à plusieurs types d'intervention. Cependant, les temps de radioscopie peuvent être différents (urétéroscopie couplée à une montée de sonde $\mathrm{JJ}$, cholédocotomie couplée à une cholécystectomie). Les regroupements doivent être validés avec l'aide des équipes des différents secteurs.

\subsection{Choix des dosimètres en vue du zonage}

Un dosimètre permettant d'évaluer l'équivalent de dose ambiant $\mathrm{H}^{*}(10)$ est nécessaire en vue de la réalisation du zonage radiologique. Plusieurs détecteurs existent et présentent des caractéristiques, des avantages et des inconvénients différents. Certains de ces détecteurs ont été comparés dans notre centre (Tableau 3).

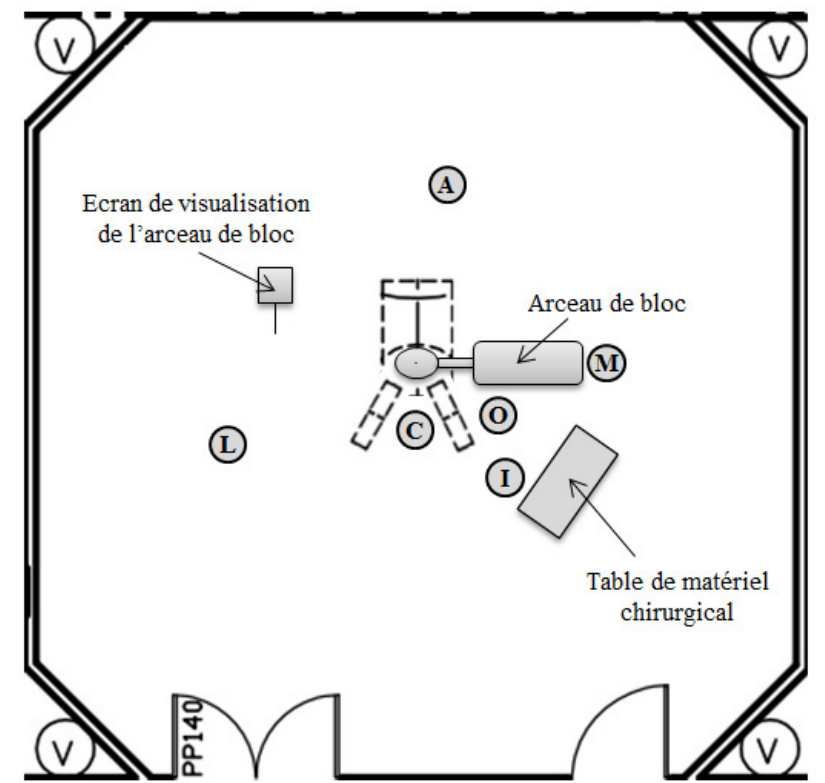

Figure 1. Plan à l'échelle d'un acte lors d'une observation. L : circulant ; A : anesthésie; C : chirurgien; I : instrumentiste ; O : aide opératoire; M : manipulateur en électroradiologie médicales. Scale drawing of an operation during observation.

Dans le cas de mesure au bloc opératoire et en l'absence de radiamètre les dosimètres opérationnels peuvent, à défaut, servir en $1^{\text {re }}$ approximation. Ce détecteur présente l'avantage de pouvoir réaliser des mesures en champs pulsés notamment inférieur à $30 \mathrm{~ms}$. Dans notre centre, après comparaison de ces différents détecteurs et travaillant uniquement en radioscopie continue, le détecteur AT11-23 ${ }^{\circledR}$ (APVL) est utilisé pour le zonage.

\subsection{Choix des dosimètres en vue des études de poste}

Pour la réalisation des études de poste de travail, les équivalents de doses individuels doivent être mesurés. Comme pour le zonage, plusieurs détecteurs existent et présentent des caractéristiques, des avantages et des inconvénients différents. Certains de ces détecteurs ont été comparés dans notre centre (Tableau 4).

Les dosimètres opérationnels ont l'avantage d'être présents en nombre suffisant sur chaque site et permettent d'évaluer à la fois $\mathrm{Hp}(10)$ et $\mathrm{Hp}(0,07)$. En l'absence de détecteur étalonné dédié, l'équivalent de dose $\mathrm{Hp}(3)$, représentatif de la dose équivalente au cristallin, est estimé par la grandeur $\mathrm{Hp}(0,07)$. Dans notre centre, après comparaison des différents détecteurs, les dosimètres opérationnels EPD Mk2 ${ }^{\circledR}$ (APVL) sont utilisés pour les études de postes.

\subsection{Modélisation des travailleurs}

Pour pouvoir matérialiser l'ensemble des personnels présents en salle, des pieds à perfusion sont positionnés à chaque position observée. Ces supports présentent l'avantage d'être légers, mobilisables facilement et permettent d'évaluer les doses délivrées pour toutes les positions en même temps. 
Tableau 3. Détecteurs comparés en vue du zonage.

Detectors compared in order to establish zoning.

\begin{tabular}{|c|c|c|c|}
\hline & $\begin{array}{c}\text { Résumé } \\
\text { des caractéristiques }\end{array}$ & Avantages & Inconvénients \\
\hline $\begin{array}{l}\text { AT11-23 } \\
\text { (APVL) }\end{array}$ & $\begin{array}{l}\text { Scintillateur plastique } \\
\text { Évalue } H^{*}(10)\end{array}$ & $\begin{array}{l}\text { Surestimation de la dose } \\
\text { en dessous de } 80 \mathrm{keV}\end{array}$ & $\begin{array}{c}\text { Pas d'intégration en champ } \\
\text { pulsé }<30 \mathrm{~ms}\end{array}$ \\
\hline $\begin{array}{l}\text { RSM } \\
\text { (Radcal) }\end{array}$ & $\begin{array}{l}\text { Chambre d'ionisation } \\
\text { Évalue } \mathrm{H}^{*}(10)\end{array}$ & $\begin{array}{l}\text { Utilisation simple } \\
\text { Intégration dans } \\
\text { un champ pulsé }\end{array}$ & $\begin{array}{l}\text { Sous-estimation de la dose } \\
\text { en dessous de } 80 \mathrm{keV}\end{array}$ \\
\hline $\begin{array}{l}10 \times 6-1800 \\
(\text { Radcal })\end{array}$ & $\begin{array}{c}\text { Chambre d'ionisation } \\
\text { Mesure un kerma dans l'air }\end{array}$ & $\begin{array}{l}\text { Détecteur de référence } \\
\text { (méthodologie IRSN) }\end{array}$ & $\begin{array}{c}\text { Coefficient de conversion } \mathrm{Gy}=>\mathrm{Sv} \\
\text { pour un spectre inconnu }\end{array}$ \\
\hline
\end{tabular}

Tableau 4. Détecteurs comparés en vue des études de poste.

Detectors compared in order to establish the occupational studies.

\begin{tabular}{|c|c|c|c|}
\hline & $\begin{array}{c}\text { Résumé } \\
\text { des caractéristiques }\end{array}$ & Avantages & Inconvénients \\
\hline $\begin{array}{l}\text { EPD Mk2 } \\
\text { (APVL) }\end{array}$ & $\begin{array}{c}\text { Diode silicium } \\
\text { Évalue } \mathrm{Hp}(0,07) \text { et } \mathrm{Hp}(10)\end{array}$ & $\begin{array}{c}\text { Nombre } \\
\text { Détecteur utilisé par les opérateurs }\end{array}$ & Temps de lecture \\
\hline $\begin{array}{l}\text { i2 } \\
\text { (RaySafe) }\end{array}$ & $\begin{array}{l}\text { Diode silicium } \\
\text { Évalue } H p(10)\end{array}$ & Nombre & $\begin{array}{c}\text { N'évalue } \\
\text { pas } \operatorname{Hp}(0,07)\end{array}$ \\
\hline $\begin{array}{l}10 \times 6-1800 \\
(\text { Radcal })\end{array}$ & $\begin{array}{c}\text { Chambre d'ionisation } \\
\text { Mesure un kerma dans l'air }\end{array}$ & $\begin{array}{l}\text { Détecteur de référence } \\
\text { (méthodologie IRSN) }\end{array}$ & $\begin{array}{c}\text { Nombre } \\
\text { Coefficient de conversion } \\
\text { Gy }=>\text { Sv pour un spectre inconnu }\end{array}$ \\
\hline $\begin{array}{l}\text { TLD-700 } \\
\text { (APVL) }\end{array}$ & $\begin{array}{l}\text { Thermoluminescence } \\
\text { Mesure une dose absorbée }\end{array}$ & $\begin{array}{l}\text { Nombre } \\
\text { Ergonomie }\end{array}$ & $\begin{array}{c}\text { Étalonnage } \\
\text { Temps de lecture } \\
\text { Coefficient de conversion } \mathrm{Gy}=>\mathrm{Sv} \\
\text { pour un spectre inconnu }\end{array}$ \\
\hline
\end{tabular}

Tableau 5. Grandeurs mesurées et estimées et hauteurs des dosimètres selon la position et la région étudiée. *Mesure réalisée derrière le tablier plombé.

Measured and estimated parameters and dosimeters' heights depending on the position and the part studied. *Measurement carried out behind lead apron.

\begin{tabular}{lcccl}
\hline Mesurée & Estimée & Hauteur $(\mathbf{m})$ & Régions & Position \\
\hline $\mathrm{Hp}(0,07)$ & $\mathrm{H}_{\text {peau }}$ & 1 & Extrémités & \\
$\mathrm{Hp}(10)$ & $\mathrm{E}$ & $1,3^{*}$ & Corps entier & Debout \\
$\mathrm{Hp}(0,07)$ & $\mathrm{H}_{\text {cristallin }}$ & 1,6 & Cristallin & \\
$\mathrm{Hp}(0,07)$ & $\mathrm{H}_{\text {peau }}$ & 1 & Extrémités & \\
$\mathrm{Hp}(10)$ & $\mathrm{E}$ & $1^{*}$ & Corps entier & Assise \\
$\mathrm{Hp}(0,07)$ & $\mathrm{H}_{\text {cristallin }}$ & 1,3 & Cristallin & \\
\hline
\end{tabular}

Par ailleurs, les doses mesurées par les dosimètres sans diffuseur sont sous-estimées (de moins de $10 \%$ ) en fonction l'angle et la distance. Trois dosimètres opérationnels sont placés face au milieu diffusant à une hauteur de 1,6 m, 1,3 m et 1,0 m pour modéliser respectivement le cristallin, le corps entier et les extrémités des différents intervenants (Tableau 5). Le dosimètre modélisant le corps entier est recouvert d'un tablier plombé. La valeur $\mathrm{Hp}(10)$ est relevée pour l'estimation de la dose efficace corps entier. La valeur $\mathrm{Hp}(0,07)$ est relevée pour l'estimation des doses équivalentes au cristallin et aux extrémités.
Pour tenir compte des situations où l'agent est en position assise, les dosimètres évaluant la dose au cristallin et au corps entier sont abaissés à $1,3 \mathrm{~m}$ et $1 \mathrm{~m}$ respectivement. Pour les actes où les chirurgiens ont les extrémités proches du faisceau (membres périphériques en traumatologie), un deuxième support, est placé plus proche du milieu diffusant.

\subsection{Modélisation du patient}

Pour tenir compte de la morphologie des patients opérés dans les différents secteurs des blocs opératoires, 4 plaques de PolyMéthylMétacrylate (PMMA) (volume total $20 \mathrm{~cm} \times$ $30 \mathrm{~cm} \times 30 \mathrm{~cm}$ ) sont utilisées. Ce volume surestime les dimensions minimales réglementaires (AFNOR, 2011). Pour reproduire les actes sur les membres (bras, jambe, main, pied, ...) des volumes d'eau équivalents ou les membres d'un fantôme anthropomorphique Rando ${ }^{\circledR}$ peuvent être utilisés.

\subsection{Choix des arceaux}

Une mesure à $1 \mathrm{~m}$ du milieu diffusant avec le même couple tension $(\mathrm{kV})$ /quantité de courant $(\mathrm{mA})$ permet de définir l'arceau de bloc le plus pénalisant. Si un type d'intervention nécessite un arceau spécifique, ce dernier sera utilisé pour les simulations. À défaut, l'arceau le plus pénalisant sera utilisé. 


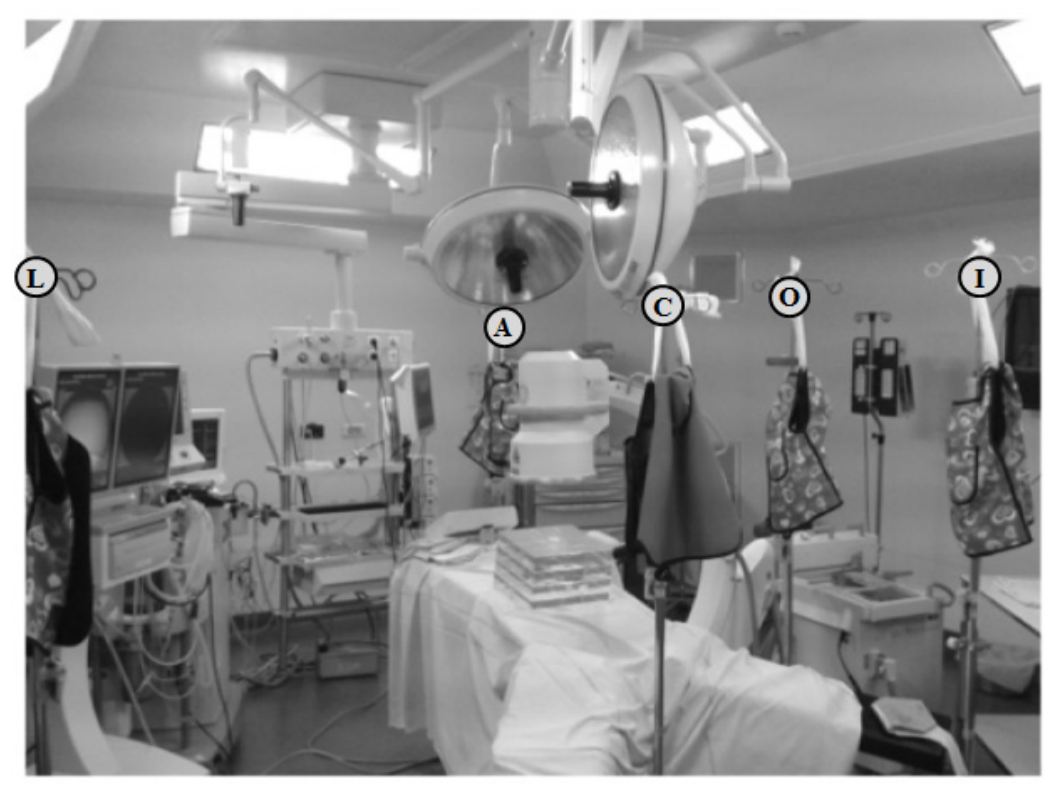

Figure 2. Disposition du matériel lors des simulations en fonction du plan réalisé lors des observations. L : circulant; A : anesthésie; C : chirurgien ; O : aide opératoire; I : instrumentiste.

Material position during the simulations according to the drawing carried out during observations.

\section{4 Étape 3 : simulations}

Les types d'interventions identifiés précédemment font l'objet de simulations afin d'évaluer les doses susceptibles d'être reçues aux différents postes de travail et de définir le zonage des locaux.

\subsection{Contrôles de la linéarité}

Pour s'assurer de la linéarité des détecteurs en fonction de la quantité de courant (mA) et du temps, les détecteurs utilisés doivent être contrôlés. Ce contrôle est réalisé en plaçant les détecteurs à $1 \mathrm{~m}$ du bord du milieu diffusant. Des acquisitions pour différents temps de mesures et différentes quantités de courant $(\mathrm{mA})$ sont réalisées.

\subsection{Choix de la disposition}

À partir du plan à l'échelle (Figure 1) réalisé lors des observations, les conditions d'exposition des différents postes de travail sont reproduites pour chaque acte simulé (Figure 2). La mesure de l'équivalent de dose ambiant est réalisée dans l'air à $1 \mathrm{~m}$ et à mi-hauteur du milieu diffusant.

\subsection{Choix des constantes}

Les tensions $(\mathrm{kV})$ et les quantités de courant $(\mathrm{mA})$ maximum de chaque type d'intervention sont moyennées et surestimées à l'entier supérieur pour les tensions $(\mathrm{kV})$ et au dixième supérieur pour les quantités de courant (mA). Les constantes sont paramétrées manuellement. Si l'intensité ne peut pas être réglée manuellement, les doses mesurées seront corrigées avec la linéarité de la quantité de courant (mA). Le mode de radioscopie le plus pénalisant est utilisé : «Scopie Continue».

\subsection{Temps de mesure et reproductibilité}

Le temps d'émission des rayonnements ionisants lors des simulations est fixé arbitrairement ( 3 minutes dans notre cas). Avec l'AT11-23 ${ }^{\circledR}$, les mesures sont réalisées en mode intégration. Pour s'assurer de la reproductibilité des mesures, toutes les simulations sont doublées.

Les doses mesurées $\left(\mathrm{D}_{\text {Simulée }}\right)$ avec les dosimètres opérationnels, exprimées en termes de $\mathrm{Hp}(10)$ ou $\mathrm{Hp}(0,07)$ selon la position simulée, seront corrigées à partir des temps de radioscopie représentatifs de chaque type d'intervention à l'étape suivante.

\section{5 Étape 4 : extrapolations}

\subsection{Extrapolation du temps de radioscopie}

Pour chaque type d'intervention, la moyenne et le troisième quartile des temps de radioscopie sont calculés. Pour majorer, la valeur la plus élevée est sélectionnée.

Les doses par acte $\left(\mathrm{D}_{\text {Acte }}\right)$ sont obtenues en extrapolant la dose simulée $\left(\mathrm{D}_{\text {Simulée }}\right)$ à la durée réelle de l'acte $(\mathrm{Eq}$. (1)).

$$
\mathrm{D}_{\text {Acte }}=\mathrm{D}_{\text {Simulée }} \times \frac{\text { Temps radioscopie }}{\text { Acte }} \text { Temps radioscopie } \text { Simulé }_{\text {Sín }} \text {. }
$$

Si la radiographie est utilisée, le nombre d'images de radiographie est traité de manière identique au temps de radioscopie (Eq. (2)).

$$
\mathrm{D}_{\text {Acte }}=\mathrm{D}_{\text {Simulée }} \times \frac{\mathrm{Nb} \mathrm{d}^{\prime} \text { images radiographie }}{\text { Acte }} \text { Nb d'images radiographie }_{\text {Simulé }} .
$$




\subsection{Extrapolation de l'activité}

Pour chaque spécialité, un certain nombre d'actes parmi les plus réalisés et/ou les plus irradiants sont sélectionnés dans la partie observations. Ces actes représentent une grande proportion (supérieure à $85 \%$ ) des actes réalisés avec rayonnements ionisants par spécialité. Cependant, la proportion manquante doit être prise en compte. Chaque acte sélectionné par spécialité $\left(\mathrm{NbA}_{\text {Sélectionné}}\right)$ va être multiplié par le rapport du nombre total d'acte de la spécialité $\left(\mathrm{NbA}_{\text {Total de la spécialité }}\right)$ sur le nombre total des actes sélectionnés pour chaque spécialité ( $\left.\mathrm{NbA}_{\text {Total sélectionné}}\right)$ (Eq. (3)).

$$
\mathrm{NbA}_{\text {Sélectionné-Extrapolé }}=\mathrm{NbA}_{\text {Sélectionné }} \times \frac{\mathrm{NbA}_{\text {Total de la spécialité }}}{\mathrm{NbA}_{\text {Total sélectionné }}} \text {. }
$$

Par exemple, en urologie, deux actes sont sélectionnés : PAC ( $\mathrm{NbA}_{\text {Sélectionné }}$ : 50$)$ et montée de JJ (NbA Sélectionné : 30). Le

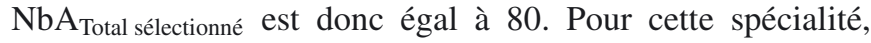

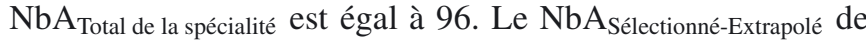
PAC est donc de 60 et le NbA Sélectionné-Extrapolé de Montée de JJ de 36. La somme du nombre d'actes sélectionnés extrapolés ( $\left.\mathrm{NbA}_{\text {Sélectionné-Extrapolé }}\right)$ correspond donc à la totalité de l'activité avec rayonnements ionisants de chaque spécialité. L'extrapolation de l'activité induit une surestimation des pratiques réelles, en accord avec le principe de précaution.

\section{6 Étape 5 : réalisation du zonage et des études de poste}

\subsection{Zonage}

Les équivalents de dose ambiant $\mathrm{H}^{*}(10)$, obtenus pour chaque type d'intervention lors des simulations, permettent de réaliser le zonage.

\subsubsection{Zonage des salles d'examens}

Pour pouvoir tenir compte de l'ensemble des actes susceptibles d'être réalisés sur une heure, nous avons fait le choix de ne pas sélectionner l'heure la plus pénalisante (DGT/ASN, 2008) mais une heure représentative de l'activité. Pour pouvoir majorer le risque, le zonage de chaque secteur est réalisé dans la salle où l'activité est la plus pénalisante et la durée de l'ensemble des actes est ramenée à 1 heure quel que soit l'acte.

L'activité est essentiellement répartie par secteur. Le zonage de chaque secteur est réalisé à partir du nombre total d'actes sélectionnés et extrapolés ( $\left.\mathrm{NbA}_{\text {Sélectionné-Extrapolé})}\right)$ de la salle la plus pénalisante.

La dose intégrée sur 1 heure $\left(\mathrm{D}_{\text {Salle/1h }}\right)$ est calculée à partir de la dose cumulée pour un acte $\left(\mathrm{D}_{\text {Acte }}\right.$ calculée à l'Eq. (1)) à l'équation (4) :

$$
\mathrm{D}_{\text {Salle/1h }}=\sum \frac{\mathrm{NbA}_{\text {Sélectionné-Extrapolé }}}{\mathrm{NbA}_{\text {Total de la spécialité }}} \times \mathrm{D}_{\text {Acte. }}
$$

Par exemple, en urologie, la $\mathrm{D}_{\text {Salle/1h }}$ serait égale à la somme du produit de la $\mathrm{D}_{\mathrm{PAC}}$ avec 62,5\% (60/96) et du produit de la D Montée JJ avec 37,5 \% (36/96).
Le nombre total d'actes avec rayonnements ionisants sur 1 mois $\left(\mathrm{NbA}_{\text {Salle/1mois }}\right)$ est déterminé pour le mois le plus pénalisant sur 12 mois glissants. La dose intégrée sur 1 mois $\left(D_{\text {Salle/1mois }}\right)$ est calculée à partir de la formule suivante (Eq. (5)).

$$
\mathrm{D}_{\text {Salle } / 1 \mathrm{mois}}=\mathrm{D}_{\text {Salle } / \mathrm{h}} \times \mathrm{NbA}_{\text {Salle } / 1 \text { mois }} .
$$

La distance entre le point de mesure et le volume diffusant (dm) est pris comme référence et les rayons des différentes zones (JORF, 2006a) sont déterminés (Eq. (6)) en fonction des valeurs des doses des différentes zones Dose(Zone) définies dans l'arrêté du 15 mai 2006.

$$
\text { Rayon }(\text { Zone })=d m \sqrt{\frac{\text { Dose intégrée à dm }}{\text { Dose (Zone })}} .
$$

La dose intégrée à $\mathrm{dm}$ (égale à $1 \mathrm{~m}$ dans notre cas) correspond à $\mathrm{D}_{\text {Salle/1h }}$ ou $\mathrm{D}_{\text {Salle/1mois }}$.

Dans le cas d'un acte rarement réalisé mais très irradiant, afin d'alerter le personnel sur le risque spécifique lié à cet acte, un deuxième zonage plus pénalisant peut être mis en place.

\subsubsection{Zonage des locaux adjacents}

Si les locaux adjacents et les couloirs sont classés en zone non réglementée, une vérification via la mise en place d'une dosimétrie passive murale (DGT/ASN, 2008) doit être effectuée. Ces dosimètres passifs sont placés à l'extérieur de la salle contre chaque paroi et relevés mensuellement. Leurs valeurs, bruit de fond déduit, doivent être inférieures à $80 \mu \mathrm{Sv}$ sur un mois pour que ces zones puissent être classées en zone non réglementée.

\section{2 Étude de poste}

Les études de poste de travail sont décomposées par spécialité et par type d'intervention. Pour chaque spécialité, pour chaque type d'intervention et pour chaque poste de travail une exposition $\left(\mathrm{D}_{\text {Acte }}\right.$ calculée à l'Eq. (1)) a été obtenue pour le corps entier, les extrémités et le cristallin. L'activité d'un agent sur une période est extrapolée (Eq. (3)). La dose cumulée ( $\left.\mathrm{D}_{\text {Cumulée }}\right)$ est obtenue par l'équation (7) :

$$
\mathrm{D}_{\text {Cumulée }}=\sum \mathrm{NbA}_{\text {Sélectionné-Extrapolé }} \times \mathrm{D}_{\text {Acte }} \text {. }
$$

Les différentes grandeurs dosimétriques utilisées, estimées et mesurées pour les études de postes selon la position sont présentes dans le Tableau 5. Ces grandeurs permettent d'établir les fiches d'exposition des différents agents en fonction de leur activité aux différents postes de travail dans les différentes spécialités en adaptant les informations disponibles aux grandeurs définies précédemment (nombre d'actes, d'heures, de jours ...). Un classement des travailleurs est alors proposé ${ }^{1}$.

Les résultats du suivi individuel de la dosimétrie opérationnelle et passive permettent de comparer les résultats théoriques

\footnotetext{
1 Articles R. 4451-44 et R. 4451-46 du code du travail.
} 
obtenus lors de l'établissement des fiches d'exposition pour l'estimation de la dose efficace avec les doses réellement mesurées. La validation des doses reçues au niveau des extrémités des opérateurs peut être réalisée via la mise en place de pastilles thermoluminescentes (TLD) à l'index en face palmaire de la main la plus proche du patient. Toutefois, l'utilisation de bagues dosimétriques engendre des problèmes d'hygiène et de confort. De plus, les doses délivrées par acte aux blocs opératoires sont globalement faibles et le cumul de dose nécessaire pour obtenir une mesure significative rend le suivi délicat.

\section{7 Étape 6 : suivi dans le temps}

\subsection{Base de données}

Les études dosimétriques sont à renouveler périodiquement et à l'occasion de toute modification des conditions pouvant affecter la santé et la sécurité des travailleurs. En pratique, cela peut se traduire par l'émergence de nouveaux actes, de changement de matériel ou de locaux. La méthodologie mise en place permet de s'adapter à l'évolution des pratiques en actualisant la base de données dosimétrique de l'activité. La mise à jour de cette base est fondamentale pour pouvoir suivre l'évolution des pratiques.

\subsection{Optimisation}

L'information, la sensibilisation et la formation des agents des différents secteurs doit être menée de manière concrète et adaptée à leurs contraintes. En effet, la radioprotection dans les blocs opératoires est souvent considérée comme accessoire au vu des risques spécifiques liés au geste invasif (aseptie, hygiène, matériel ...). Toutefois, l'application de principes de radioprotection simples (distance-temps-écran) permet de réduire fortement l'exposition des différents agents, dès lors qu'ils ont été sensibilisés par la PCR. De plus, la mise en place de la base de données dosimétriques aux blocs opératoires permet au fil du temps de faciliter l'optimisation des pratiques en lien avec la PSRPM. Cette optimisation est basée sur la diminution de la dose patient qui implique une réduction de dose pour chaque intervenant. Le travail conjoint de la PCR et de la PSRPM est donc indispensable pour une optimisation globale de la radioprotection dans ce secteur où la radioprotection des patients et des travailleurs sont intimement liées.

\section{Conclusions}

La mise en place de la radioprotection aux blocs opératoires est longue et complexe. La méthodologie proposée, composée de 6 étapes, est applicable à tous les secteurs où sont effectués des actes médicaux guidés par radioscopie. Elle permet de pallier les difficultés rencontrées afin de réaliser les études de poste et le zonage. Une attention régulière de la PCR est nécessaire pour éviter les dérives et former les nouveaux personnels dans ces secteurs. Un regroupement des activités, nécessitant des rayonnements ionisants sur des salles dédiées, permettrait de faciliter la gestion des risques et d'encadrer les pratiques.

\section{Références}

AFNOR (2011) Norme NF C 15-160 Installations pour la production et l'utilisation de rayonnements X - Éxigences de radioprotection.

DGT/ASN (2008) Circulaire DGT/ASN n 1 du 18 janvier 2008, relative à l'arrêté du 15 mai 2006 relatif aux conditions de délimitation et de signalisation des zones surveillées et contrôlées et des zones spécialement réglementées ou interdites compte tenu de l'exposition aux rayonnements ionisants, ainsi qu' aux règles d'hygiène, de sécurité et d'entretien qui y sont apposées.

IRSN (2010) Guide pratique : Réalisation des études dosimétriques de poste de travail présentant un risque d'exposition aux rayonnements ionisants (version 2), DRPH/DIR n 2010-1.

JORF (2003) Décret n 2003-296 du 31 mars 2003 relatif à la protection des travailleurs contre les dangers des rayonnements ionisants, Journal Officiel de la République Française du 2 avril 2003.

JORF (2006a) Arrêté du 15 mai 2006 modifié relatif aux conditions de délimitation et de signalisation des zones surveillées et contrôlées et des zones spécialement réglementées ou interdites compte tenu de l'exposition aux rayonnements ionisants, ainsi qu'aux règles d'hygiène, de sécurité et d'entretien qui y sont imposées, Journal Officiel de la République Française du 15 juin 2006.

JORF (2006b) Arrêté du 22 septembre 2006 relatif aux informations dosimétriques devant figurer dans un compte rendu d'acte utilisant les rayonnements ionisants, Journal Officiel de la République Française du 29 septembre 2006.

JORF (2011) Arrêté du 6 décembre 2011 relatif à la formation et aux missions de la personne spécialisée en radiophysique médicale et à la reconnaissance des qualifications professionnelles des ressortissants étrangers pour l'exercice de ces missions en France, Journal Officiel de la République Française du 18 décembre 2011.

Schiedts D. (2014) Le bloc : cancre de la radioprotection? Dans : Sixièmes journées sur l'optimisation de la radioprotection dans les domaines nucléaire, industriel et médical, SFRP, Saint-Malo.

Cite this article as: C. Duverger, G. Moliner, F. Boury, P. Branchereau, P. Costa, J.P. Beregi, J. Greffier. Étude de poste et zonage aux blocs opératoires : méthodologie pratique en six étapes. Radioprotection 50(4), 287-293 (2015). 